\title{
PRODUCTION, TRAITEMENT ET COMMERCIALISATION \\ DU LAIT DE CONSOMMATION EN NATURE (1)
}

\author{
par \\ G. THIEULIN
}

Le lait de consommation en nature, e'est-à-dire pouvant être consommé directement en l'état où il est présenté, admet actuellement quatre formes : cru, pasteurisé, stérilisé, reconstitué, étant, dans ce dernier cas, tel quel ou pasteurisé ou stérilisé.

Ce peut être, finalement, du lait entier ou du lait partiellement ou totalement écrémé.

Enfin, s'il n'est pas totalement écrémé, il peut être homogénéisé.

\section{Production}

A ce stade, nous distinguons deux cas :

a) Production d'un lait destiné à être consommé directement à l'état cru.

Il s'agit, de toute évidence, d'un produit exceptionnel, de haute qualité, caractérisé par un prix de revient fatalement très élevé en raison des garanties sanitaires à rechercher sans le secours de l'assainissement thermique, celles-ci ne pouvant être présumées atteintes que sous condition de prendre des précautions extrêmement sévères sur lesquelles nous n'avons pas, dans ce bref développement, à insister.

En conséquence, un tel lait ne saurait intéresser qu'un nombre très restreint de consommateurs et ne doit pas retenir davantage ici notre attention.

b) Production d'un lait destiné à être pasteurisé, ou stérilisé, ou déshydraté pour être ultérieurement reconstitué.

Tout en exigeant, pour base indispensable, le bon état sanitaire et une alimentation à la fois suffisante et équilibrée des femelles laitières, il convient de réaliser simplement, avec un prix de revient normal, des conditions de production satisfaisantes du point de vue

(1) Communication présentée au $3^{\mathrm{e}}$ Symposium international de Zootechnie, le 15 avril 1968, à Milan (Italie). 
de l'hygiène (propreté de la traite et des manipulations, emploi désirable de la réfrigération, livraison rapide au Centre de traitement thermique).

\section{Traitement et commercialisation}

A) Pour le lait pouvant être consommé à l'état cru, rappelons, entre autres servitudes, la nécessité d'une réfrigération énergique dès la traite, d'un conditionnement rapide, puis du maintien à basse température, et enfin d'un délai de commercialisation ne dépassant pas normalement 24 heures. Restant à souligner que du point de vue de l'hygiène alimentaire, un tel lait, même si la sécurité bactériologique était obtenue, présentera toujours certains inconvénients du point de vue diététique, ne serait-ce que par sa teneur inconstante en matière grasse.

B) Pour le besoin universel des populations, en particulier de celles des agglomérations urbaines, nous retiendrons d'abord, objectivement, le lait pasteurisé.

\section{Le lait pasteurisé}

Le traitement thermique considéré doit concerner, non plus le lait cru exceptionnel dont nous venons de parler mais, au contraire, un lait cru de production courante, révélant une qualité hygiénique suffisante pour que l'on puisse aboutir, à la suite de l'action thermique rationnellement appliquée, aux qualités requises.

Un tel lait cru peut être défini par l'heureuse expression de C. Gorini : "lait de pasteurisation facile».

La pasteurisation peut alors, avec grand profit, être effectuée selon la technique dite "High Temperature Short Time ", e'est-àdire à $75^{\circ}$ pendant 15 à 16 secondes, afin de conserver au lait ses qualités biologiques d'origine et de lui conférer la sécurité nécessaire sur le plan bactériologique, sans provoquer l'apparition de saveurs indésirables.

Un conditionnement réalisé, en unités de vente au détail, immédiatement après le refroidissement, lui-même consécutif au chauffage, puis le maintien du lait à $3-4^{\circ} \mathrm{C}$, facilitent une commercialisation dont le délai offrira une assez grande latitude, principalement dans le cas où le lait aura été homogénéisé, se trouvant ainsi physiquement stabilisé.

Cependant, l'heureuse évolution des conditions de vie, entrầnant un repos hebdomadaire d'une et, peut-être bientôt, de deux journées consécutives, la nécessité d'assouplir les circuits commerciaux en diminuant la fréquence des livraisons de l'usine aux détaillants et en les limitant à certaines heures, nous conduit à 
une orientation nouvelle d'autant plus valable que les progrès réalisés en technologie laitière nous la permettent aisément.

Ainsi, en faveur d'un large et facile approvisionnement des diverses populations du globe en lait de consommation en nature, il convient de porter notre attention sur :

- Le lait stérilisé.

- Le lait reconstitué à partir d'un lait déshydraté.

\section{Le lait stérilisé}

Comme pour le lait pasteurisé, et d'ailleurs pour tout autre lait destiné à l'alimentation humaine, le lait stérilisé doit être préparé à partir d'un lait cru de bonne qualité hygiénique. En aucun cas l'action thermique, même si elle correspond à la stérilisation, ne permet d'aboutir à un résultat favorable si elle est appliquée à un lait souillé ou de composition défectueuse. C'est là un principe fondamental qu'il convient de rappeler sans cesse.

Soulignons à nouveau notre objectif quant aux caractéristiques que doit présenter le lait de consommation en nature : valeur biologique, sécurité bactériologique, caractères organoleptiques favorables, stabilité commerciale.

Les deux formules ci-dessus évoquées peuvent répondre à cette quadruple exigence.

Tablant sur un niveau initial valable, par l'état sanitaire des vaches laitières et la qualité de leur alimentation ainsi que par une hygiène générale économiquement appliquée, il reste à faire subir au lait cru - matière première livrée par le producteur - un traitement qui maintienne ses qualités initiales, qui rende constante sa teneur en matière grasse (notion importante en diététique), qui interdise ou détruise toute contamination bactérienne, qui aboutisse à des caractères organoleptiques incitant à une consommation étendue et à une bonne assimilation, et qui, enfin, permette une commercialisation facile.

Le lait pasteurisé ne peut pas répondre à cet ensemble, ne seraitce que par les difficultés de son transport et la faible durée de sa conservation.

Nous sommes alors amené à apprécier les moyens technologiques, qui sont aujourd'hui à notre portée, pour atteindre le but fixé.

Les procédés classiques de stérilisation ne nous donnent pas satisfaction (stérilité souvent illusoire, goût "de cuit " plus ou moins prononcé, altérations biochimiques). Par ailleurs, le conditionnement en verre transparent, d'un lait homogénéisé, donc très sensible à l'action de la lumière, entraîne, soit une-servitude commerciale par la protection qui s'impose contre les rayons lumineux, soit une dégradation des caractères organoleptiques. 
Par contre, le lait chauffé à très haute température (140-150 C) pendant un temps très court (ordre de grandeur de la seconde), procédé général dit U.H.T., représente indiscutablement un excellent moyen de parvenir aux fins précisées, d'autant plus que le conditionnement "aseptique » en unités de vente au détail est devenu réalité et que nous disposons aisément de conditionnements opaques à la lumière, hermétiquement scellés.

Si les installations en place ne permettent pas, du point de vue économique, de généraliser rapidement ce nouveau procédé, quelles qu'en soient les variantes en pratique, il n'en est pas moins juste de prétendre qu'il existe, d'en proclamer la grande valeur, et d'affirmer que, grâce à lui, le problème posé se trouve, malgré sa complexité, convenablement résolu.

Et nous passons à la dernière formule : le lait reconstitué à partir d'un lait déshydraté.

\section{Le lait reconstitué à partir d'un lait déshydraté}

Il n'est évidemment question que d'une reconstitution industrielle, parfaitement réglée, en vue d'un lait stérilisé analogue au précédent, permettant, notamment, l'approvisionnement de régions plus ou moins dépourvues de production laitière.

Les pays de climat tempéré, producteurs excédentaires, ont alors la possibilité d'expédier à grande distance un lait déshydraté, la reconstitution sur place offrant ensuite, sous toutes les garanties d'hygiène désirées, à des populations carencées en protéines, un aliment de premier ordre dont la valeur alimentaire sera constante et sciemment déterminée.

Quatre variétés de préparation sont à envisager :

- Lait déshydraté, entier ou écrémé, reconstitué tel quel.

- Lait déshydraté, écrémé, reconstitué avec addition de ma. tière grasse butyrique ou huile de beurre (= lait recombiné).

- Lait déshydraté, entier ou écrémé, reconstitué en étant mélangé à du lait indigène (lait coupé = toned milk), cette formule utilisant et favorisant la production locale.

- Lait déshydraté, écrémé, reconstitué avec enrichissement à l'aide d'une matière grasse étrangère (végétale), de production locale abondante et bon marché (lait imitation $=$ filled milk).

Ajoutons la possibilité de préparer - contre la carence protéique - un lait enrichi en protéines.

Nous ne saurions, dans ce bref exposé, entrer dans les détails de telles opérations à l'échelle mondiale, mais, déjà, d'heureuses réalisations peuvent servir d'exemples.

\section{Conclusion}

Le lait pasteurisé, le lait stérilisé, le lait reconstitué à partir d'un lait déshydraté, représentent, pratiquement, les trois formules actuelles du lait de consommation en nature. 
Les critères énumérés pour que ces différents laits soient satisfaisants, du point de vue de l'hygiène, du point de vue économique et sur le plan social, peuvent être aisément atteints grâce aux moyens technologiques dont nous disposons maintenant.

Cependant, au stade initial, doivent être impérativement résolus les problèmes liés à l'élevage rationnel des femelles laitières, à leur alimentation suffisante et équilibrée, à leur état sanitaire, ainsi qu'aux mesures d'hygiène générale intéressant la production du lait.

Tout ceci montre l'extrême intérêt et le choix combien judicieux des sujets portés au programme de ces Journées d'étude.

\section{Summary}

Different varieties of wholesome, with good flacour, market milk can be offered to consumers :

- Raw milk, of superior quality only, and then with very high net costs.

- Pasteurized milk H.T.S.T.

- Sterilized milk U.H.T.

- Recombined milk, toned milk, filled milk, and, if necessary, protein increased milk. 